\title{
TEMPLATE MATCHING TECHNIQUE FOR SEARCHING WORDS IN DOCUMENT IMAGES
}

\author{
Dr. S.Vijayarani ${ }^{\#}$ and Ms. A.Sakila* \\ Assistant Professor ${ }^{\#}$,Department of Computer Science, School of Computer Science \\ and Engineering, BharathiarUniversity, Coimbatore \\ M.Phil Research Scholar ${ }^{*}$ Department of Computer Science, School of Computer \\ Science and Engineering, BharathiarUniversity, Coimbatore
}

\begin{abstract}
Template matching technique is useful for searching and finding the location of a template image (Small part of image) in the larger image. This technique is also used in Optical Character Recognition (OCR) tools and these tools are used for converting the scanned document images into normal text. Template matching technique is used to find and recognize the template image which is found in the given input image. In this research work, template matching technique is applied for scanned document images which contains characters (both uppercase and lowercase) and numerals. In order to perform the comparison of the template image with the input image we have used Performance Index method and it is compared with the normalized cross correlation and cross correlation methods. Different types of comparisons done in this work are, (i) comparing single character from a word, sentence and paragraph; (ii) comparing multiple characters (words) from a word, sentence and paragraph.
\end{abstract}

\section{KEYWORDS}

Document image, OCR, Template Matching, Fixed Size Templates and Performance Index Method.

\section{INTRODUCTION}

Optical Character Recognition (OCR) is used to convert the given input image in to text form i.e. normal document form in which we can able to do all the editing tasks [1].In addition to this, OCR tools retrieved the text from the images by applying template matching technique. Template matching is a technique used in digital image processing for finding small parts of an image which matches a template image [6] and it is commonly used for processing images and pictures [8]. Based on the template image, (i.e. search element) it performs the searching process in the input image and it identifies the location of the search element. Template matching is classified into two approaches; they are Feature-based approaches and Template or Area based approaches.

A feature in feature-based approach includes curves, points and surfaces. The main aim is to find the relationship between the search image and template image using the spatial or feature descriptors. Spatial relations, invariant descriptors, pyramids, wavelets and relaxation methods are sub category of feature based approach [8].Area based approach is sometimes called as correlation-like methods or template matching. This method is best suited for the templates which have no strong features with image; they operate directly with large number of values. Matches are based on the intensity values of both input image and template image. Squared

DOI: $10.5121 /$ ijci.2015.4603 
differences in fixed intensities, correction-based methods, optimization methods and mutual information are some of the area-based approaches [8].

The primary objective of this work is to identify the location of the given template character image in the scanned document images. This searching process is performed by the template matching technique which uses performance index method.

The remaining portion of this paper is discussed as follows. Section 2 discusses the related works. Section 3 provides the Methodology. Section 4 gives the output results. Conclusion is given in Section 5.

\section{RELATED WORK}

Chin-Sheng Chen,et.al [2] has analyzed the template matching techniques using statistical models and parametric template for multi-template. This algorithm contains of two phases such as training and matching. In the training phase, the statistical model created by principal component analysis method can be used to create multi-template. In the matching phase, the normalized cross correlation is used to find the part of an image. They proposed a template matching algorithm based on multi-template using training and matching phases [2]. Image block and multi-template is built to use the parametric template method.

KavitaAhuja,et.al [8] have analyzed the performance of two template matching algorithm and these algorithms used the correlation method and phase angle method to recognize the object. The goal is to find similar objects when input is entirely in image form. Phase angle method takes very few seconds to recognize the objects in images. On rotating same images, correlation method takes less time for recognizing the same objects.

Ankit Kumar,et.al [16] has implemented an approach which focused on the core basics of the template matching in remote sensing images and using two approaches SAD (sum of absolute differences) and SSD (sum of squared differences), template matching for finding the object. This work finds the GCP (ground control point) and generated the template forms and these templates can run on the image and to find the correct location of the temporal image. With this approach, it is possible to count the object and access the object condition.

Duc Thanh Nguyen et.al. [25] has proposed a novel template matching method known as Chamfer matching method. It manipulates a generalized distance transform (GDT) and an orientation map (OM). The GDT allows weighting the distance transform more on the strong edge points. And OM provides added local orientation information for matching. Two stages of human detection method such as template matching and Bayesian verification has been developed. This method can effectively reduce the false positive and false negative detection rate. The proposed algorithm conventional Chamfer matching method significantly improved the detection performance.

\section{METHODOLOGY}

\subsection{Input Image}

Input image (text image) is captured using electronic devices such as optical (digital/video) camera; scanner and mobile phones [3]. The images will be stored as image file formats like JPG/JPEG, BMP, PNG, TIFF, GIF etc. The character images are detected from the template string. 


\subsection{Convert to Binary Images}

The process of converting the color image into black and white image is called a binary image. This method is based on various color transforms. According to the R, G, B value in the image, it calculates the values of grey scale and also obtains the grey image [3].Template matching technique can be easily performed on grey images or edge images [7].

\subsection{Find Character Boundaries}

This step finds the character boundaries by using template image. Template image is a small portion of an input image; it is used to find the template in the given search image. Template matching technique is used to find character boundaries. The work flow of the template matching is illustrated in figure1.

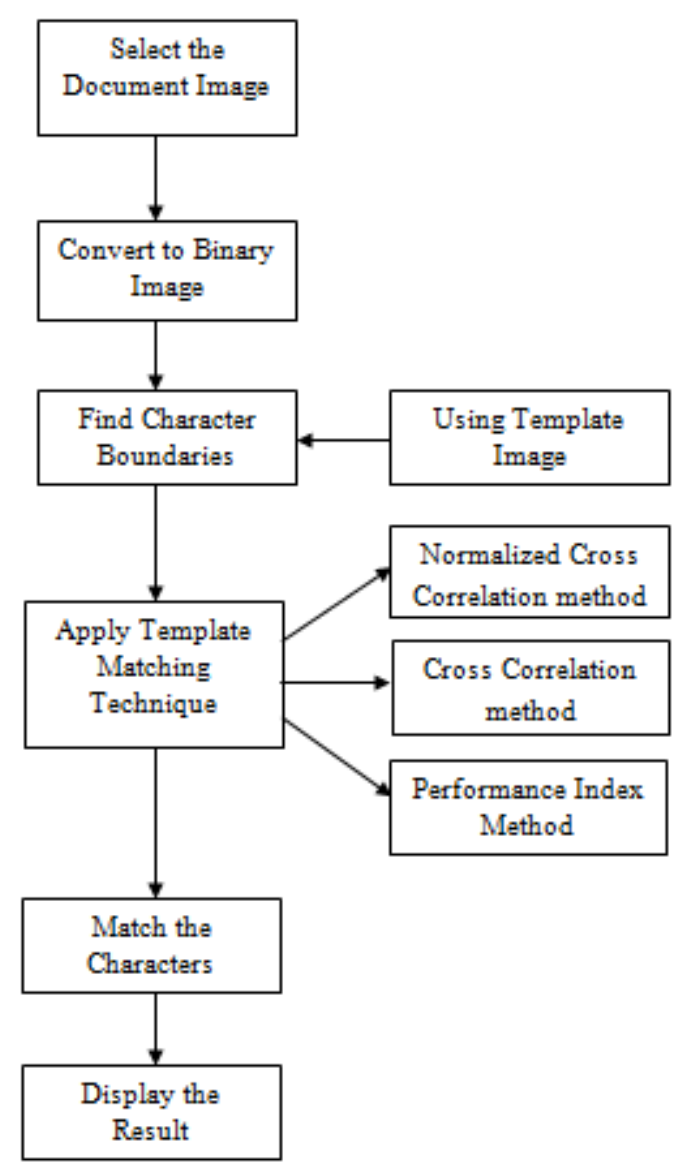

Figure1. Workflow of the Template matching

\subsection{Template Matching}

Template matching technique can be applied only with the help of template images. It is used to find the pixel level and matches the character boundaries in the template image. Many 
template matching techniques are used in image processing, which are used to find the location of the search image.

\subsubsection{Cross Correlation}

The cross-correlation template matching is motivated by the distance measure (squared Euclidean distance) [17], [18]

$$
d_{f, t}^{2}(u, v)=\sum_{x, y}[f(x, y)-t(x-u, y-v)]^{2}
$$

Equ.1

Where $\mathrm{f}$ is the input image and $\mathrm{t}$ is the template image, the sum is over $\mathrm{x}, \mathrm{y}$ under the window containing the feature $t$ positioned at $(\mathrm{u}, \mathrm{v})$. In the expansion of $\mathrm{d}^{2}$

$$
d_{f, t}^{2}(u, v)=\sum_{x, y}\left[f^{2}(x, y)-2 f(x, y) t(x-u, y-v)+t^{2}(x-u, y-v)\right]
$$

Equ.2

The term $\sum \mathrm{t}^{2}(\mathrm{x}-\mathrm{u}, \mathrm{y}-\mathrm{v})$ is constant. If the term $\sum \mathrm{f}^{2}(\mathrm{x}, \mathrm{y})$ is approximately constant, then the remaining cross-correlation term.

$$
c(u, v)=\sum_{x, y} f(x, y) t(x-u, y-v)
$$

Equ.3

is a measure of the similarity between the image and the feature.

\subsubsection{Normalized Cross Correlation}

There are several disadvantages for using the Equ.3 for template matching [19][24]

- If the image energy varies with position $\sum \mathrm{f}^{2}(\mathrm{x}, \mathrm{y})$ matching using equ. 3 can fail. For example, the correlation between feature and an exactly matching region in the image may be less than the correlation between the feature and a bright spot [24].

- $\quad$ The range of $\mathrm{c}(\mathrm{u}, \mathrm{v})$ is dependent on the size of the feature.

- $\quad$ Equ.1 is not invariant to changes in image amplitude such as those caused by changing lighting conditions across the image sequence.

The correlation coefficient overcomes these difficulties by normalizing the image and feature vectors to unit length, yielding a cosine-like correlation coefficient [19]. 


$$
\gamma=\frac{\sum_{x, y \sqrt{ }}\left(f(x, y)-\bar{f}_{u, v}\right)(t(x-u, y-v)-\bar{t})}{\sqrt{\sum_{x, y}\left(f(x, y)-\bar{f}_{u, v}\right)^{2}} \sum_{x, y}(t(x-u, y-v)-\bar{t})^{2}}
$$

Equ.4

is the mean of the feature and is the mean of $\mathrm{f}(\mathrm{x}, \mathrm{y})$ in the region under the feature. We refer to Equ. 4 as Normalized Cross-Correlation.

\subsubsection{Performance Index Method}

$\mathrm{S}\left(\mathrm{x}_{\mathrm{s}}, \mathrm{y}_{\mathrm{s}}, \mathrm{z}_{\mathrm{s}}\right)$ represents the coordinates of each pixel in the input image (source image). $\mathrm{T}\left(\mathrm{x}_{\mathrm{t}}\right.$, $\left.\mathrm{y}_{\mathrm{t}}, \mathrm{z}_{\mathrm{t}}\right)$ are coordinates of each pixel in the template image (search element). Then move the center (or the origin) of the template $\mathrm{T}\left(\mathrm{x}_{\mathrm{t}}, \mathrm{y}_{\mathrm{t}}, \mathrm{z}_{\mathrm{t}}\right)$ over each $\left(\mathrm{x}_{\mathrm{s}}, \mathrm{y}_{\mathrm{s}}, \mathrm{z}_{\mathrm{s}}\right)$ point in the input image and calculate the sum of products between the coefficients in $S\left(x_{s}, y_{s}, z_{s}\right)$ and $T\left(x_{t}, y_{t}, z_{t}\right)$ over the whole area spanned by the template. The position of the template image with respect to the input image is measured. Equ. 5 SAD (Sum of absolute differences) is a measure of template matching used to compare the intensities of the pixels in the image [7].

A pixel in the input image with coordinates $\left(x_{s}, y_{s}, z_{s}\right)$ has intensity $I_{s}\left(x_{s}, y_{s}, z_{s}\right)$ and a pixel in the template with coordinates $\left(\mathrm{x}_{t}, \mathrm{y}_{\mathrm{t}}, \mathrm{z}_{\mathrm{t}}\right)$ has intensity $\mathrm{I}_{\mathrm{t}}\left(\mathrm{x}_{\mathrm{t}}, \mathrm{y}_{\mathrm{t}}, \mathrm{z}_{\mathrm{t}}\right)$. Thus the absolute difference $(\mathrm{SAD})$ in the pixel intensities is defined as $\operatorname{Diff}\left(\mathrm{x}_{\mathrm{s}}, \mathrm{y}_{\mathrm{s}}, \mathrm{z}_{\mathrm{s}}\right.$ and $\left.\mathrm{x}_{\mathrm{t}}, \mathrm{y}_{\mathrm{t}}, \mathrm{z}_{\mathrm{t}}\right)=\mid \mathrm{I}_{\mathrm{s}}\left(\mathrm{x}_{\mathrm{s}}, \mathrm{y}_{\mathrm{s}}, \mathrm{z}_{\mathrm{s}}\right)-$ $\mathrm{I}_{\mathrm{t}}\left(\mathrm{x}_{\mathrm{t}}, \mathrm{y}_{\mathrm{t}}, \mathrm{z}_{\mathrm{t}}\right) \mid$

$$
\operatorname{SAD}(x, y, z)=\sum_{i=0}^{\text {Trows }} \sum_{j=0} \sum_{z=0}^{\text {TcolsTrowstcols }} \operatorname{Diff}(x+i, y+j, z+(i, j))
$$

Equ.5

The mathematical representation of the pixels in the input image translates the origin of the template at every pixel and takes the SAD measure which is as follows.

$$
\sum_{i=0}^{\text {Srows ScolsSrowstcols }} \sum_{j=0} \sum_{z=0} \operatorname{SAD}(x, y, z)
$$

Equ.6 Equ. $6 S_{\text {rows }}$, $S_{\text {cols }}$ denotes the rows, columns and $S_{\text {rows }+ \text { Cols }}$ stores the value of rows and columns of the Input image and $\mathrm{T}_{\text {rows }}, \mathrm{T}_{\text {cols }}$ denotes the rows, columns and $\mathrm{T}_{\text {rows }}+$ Cols stores a value of rows and columns of the template image, respectively. The lowest SAD score gives the measurement for the best position of template image within the input image.

\subsection{Match the characters}

This step matches the characters in Template matching. In this work Performance Index method is used to find the location of template image in the source image. 


\section{Pseudo code for Performance Index Method}

Step1: Select the template search element from the source input image.

Step2: Load the input image and the template image.

Step3: Compute SAD by moving the template image over the input document image. Three variables $\mathrm{x}, \mathrm{y}, \mathrm{z}$ are used to store and check the pixel size.

Step4: Image is checked based on the pixel size of rows and columns using $\mathrm{x}, \mathrm{y}$ variables and $\mathrm{z}$ variable store the rows and columns.

Step5: for loop is used to check the template matching.

Step6: Fixing threshold value for template image matching.

Step7: Template is not found in the input image then go to step 3 else,

Step8: If template is found in the input image then display the result.

\subsection{Display the Result}

The search image found in the input image is displayed.

\section{RESULT OF TEMPLATE MATCHING}

In this work three template matching techniques namely cross correlation, normalized cross correlation and performance index method are compared. This analysis is performed in MATLAB tool.

\section{A. CROSS-CORRELATION}

Cross-Correlation has commonly used between two inputs and it is a one kind of template matching [20]. The template matching result of Cross correlation method is shown in Figure 2.
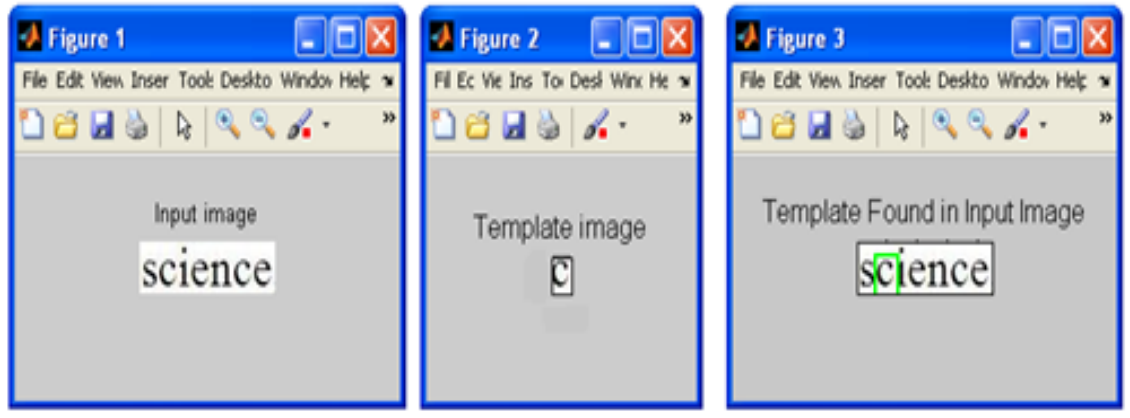

Figure 2. Cross Correlation

\section{B. NORMALIZED CROSS CORRELATION}

The template matching result using Normalized cross correlation method is shown in Figure 3. 

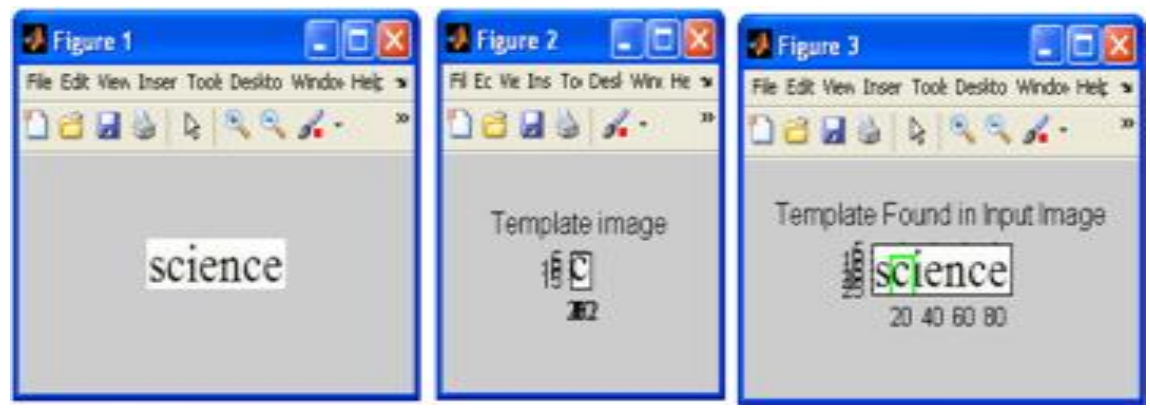

Figure 3. Normalized Cross Correlation

\section{PERFORMANCE INDEX METHOD}

Template matching using performance index method provides accurate results. Two types of comparisons are done in this method (i) comparing single character from a word, sentence and paragraph; (ii) comparing multiple characters (words) from a word, sentence and paragraph.

\subsection{Single letter match in a Single Word}

The single letter match in a single word is nothing but to match a single letter in a single word Figure 4.1 displays the input image and Figure 4.2 shows template image which is used to find the location and matches the single letter in the input image. The results of the proposed method is given in Figure 4.3

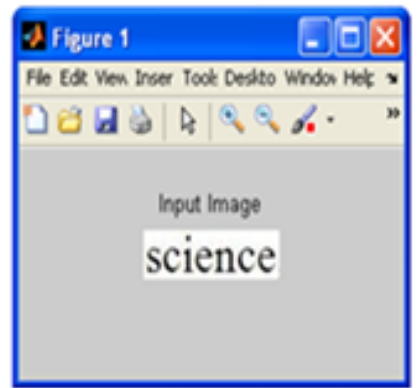

Fig 4.1 Input image

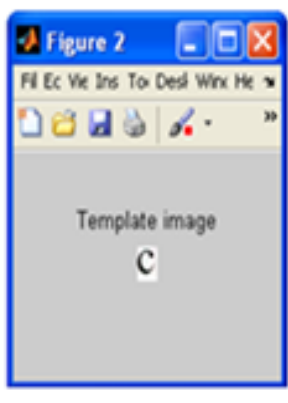

Fig 4.2 Template

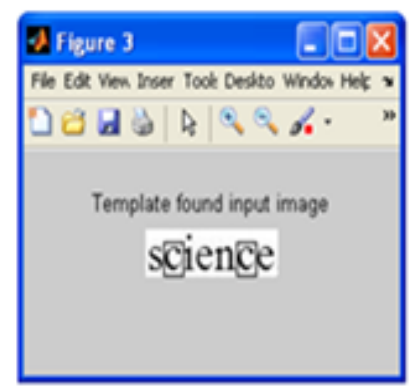

Fig 4.3 Result

\subsection{Single letter match in a Sentence}

In this strategy, we have to verify whether the single letter is found in the paragraph or not. Figure 5.1 shows an input image and Figure 5.2 displays the template image. Figure 5.3 provided the search results. 


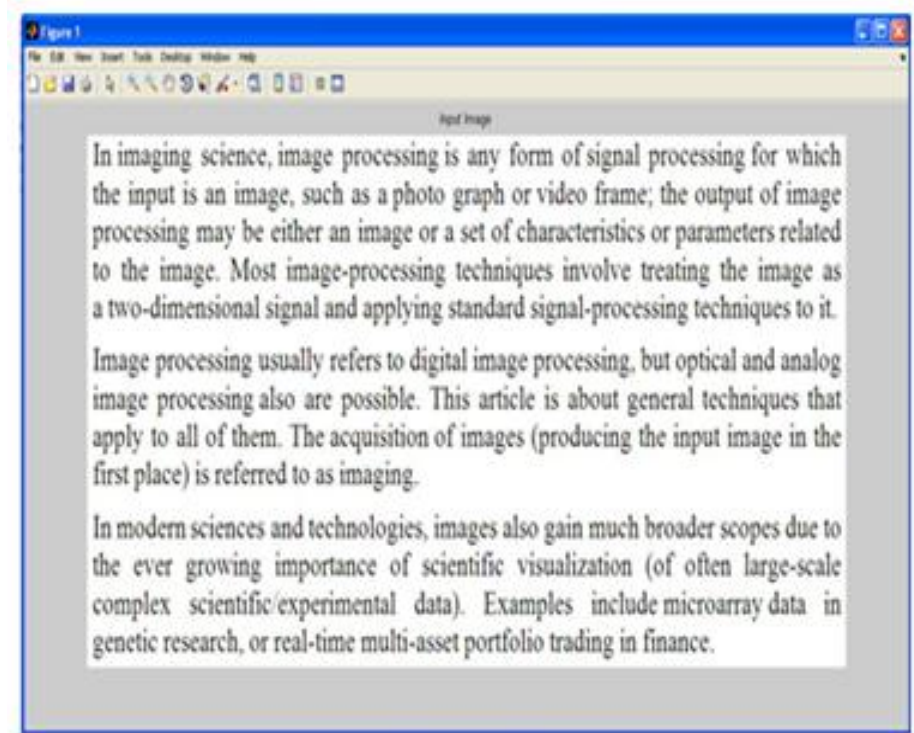

Fig 5.1 Input image

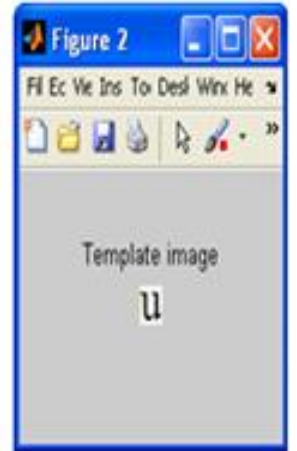

Fig 5.2 Template

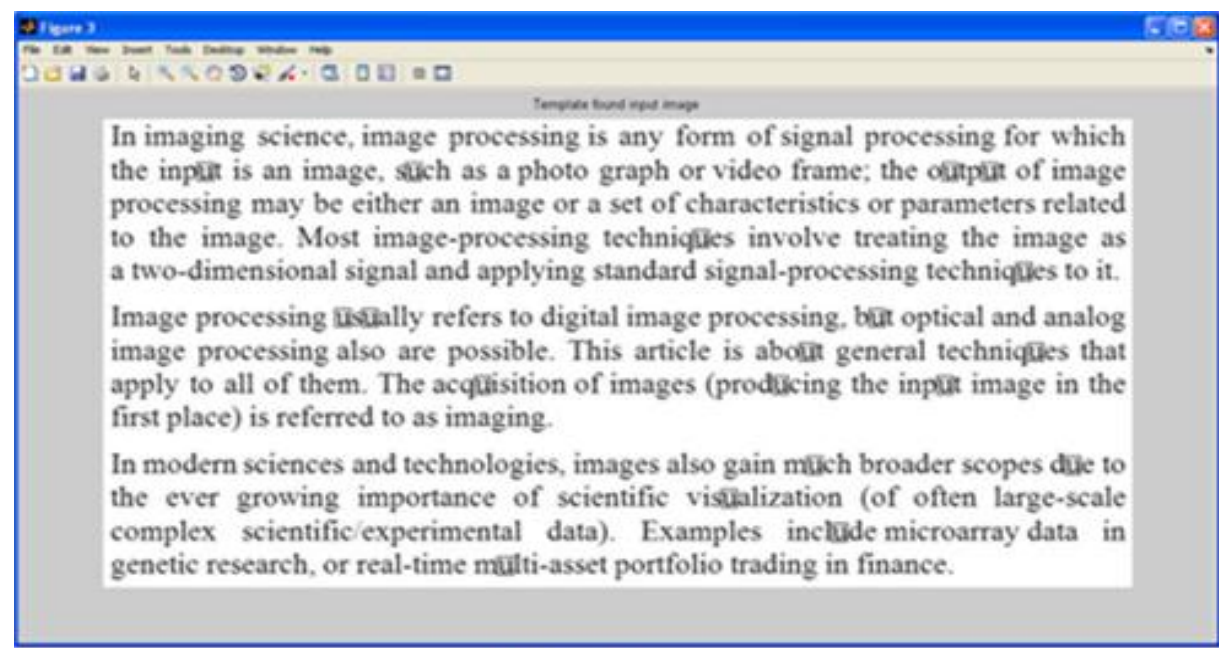

Fig 5.3 Result

\subsection{Multiple Character match in a single word}

In this multiple character match, we have to verify whether the given string is found in the word or not. Figure 6.1 shows a input image and Figure 6.2 displays the template image and the resultant image is given in Figure 6.3

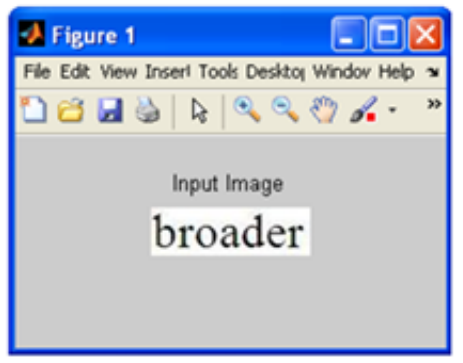

Fig 6.1 Input image

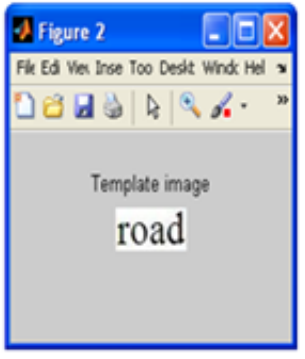

Fig 6.2 Template

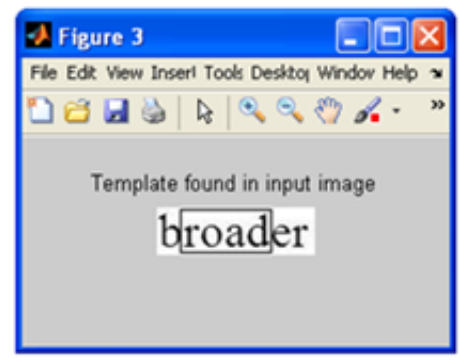

Fig 6.3 Result 


\subsection{Multiple Character match in a Paragraph}

The multiple characters match the paragraph in which we have to verify whether the given string is found or not. Figure 7.1 shows a input image and Figure 7.2 displays the template image and the resultant image is given in Figure 7.3

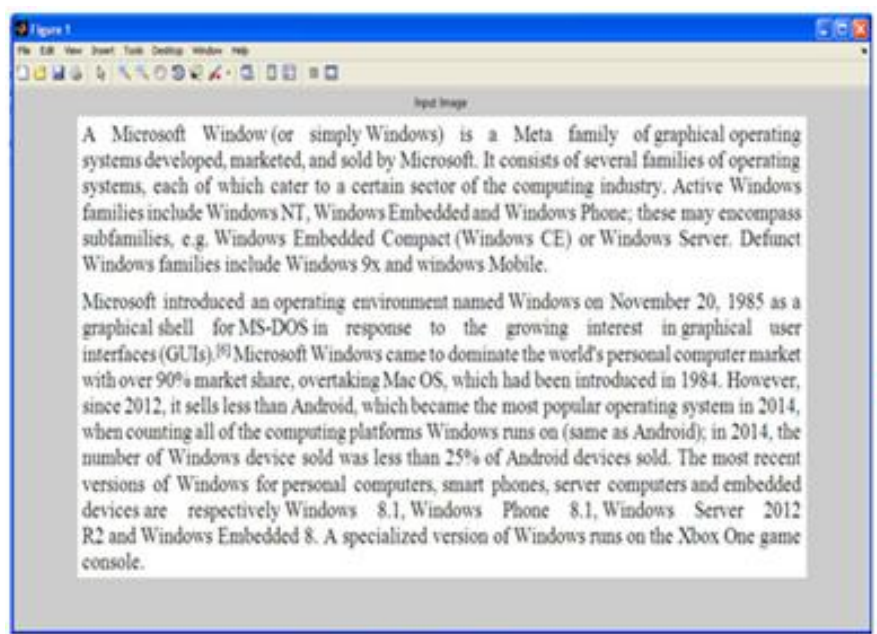

Fig 7.1 Input image

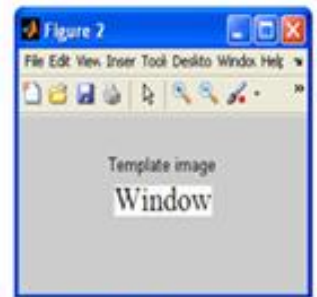

Fig 7.2 Template

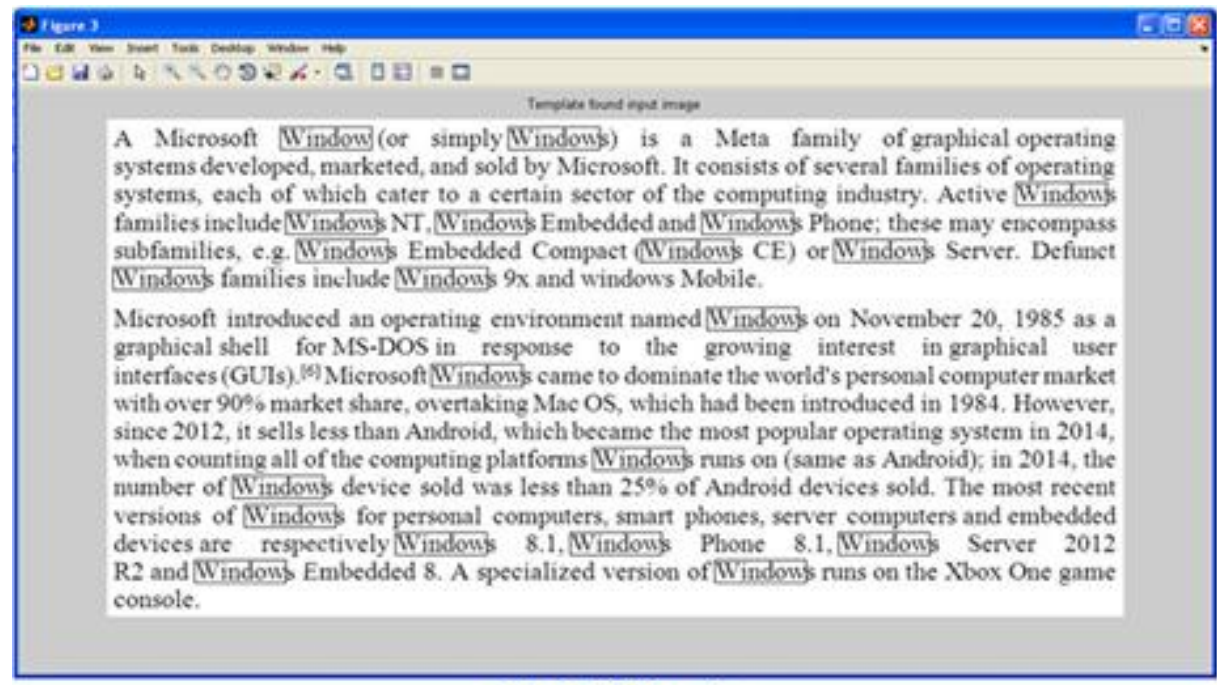

Fig 7.3 Result

\section{CONCLUSION}

This work has analyzed with three template matching techniques namely normalized cross correlation, correlation methods and performance index method. The observation of this work is that the performance index method has better accuracy compared with normalized cross correlation and correlation methods. From this analysis, we come to know that this performance index method works with same size and font of the template image in the input image. Whereas performance index methods give accurate result for the document images. Many challenging research problems are available in scanned document images. These problems can be solved by developing new algorithms, concepts and techniques. The future 
work can be analyzed and implemented with different image sizes and fonts with newly proposed algorithm.

\section{REFERENCE}

[1] SameekshaBarve, "Optical Character Recognition Using Artificial Neural Network" International Journal of Advanced Research in Computer Engineering \& Technology, Volume 1, Issue 4, June 2012, pp.131-133

[2] Chin-Sheng Chen, Jian-Jhe Huang, Chien-Liang Huang, "Template Matching using Statistical Model and Parametric Template for Multi-Template'Journal of Signal and Information Processing, August 2013, pp.52-57

[3] Mr. G.T. Sutar, Prof. Mr. A.V. Shah, "Number Plate Recognition Using an ImprovedSegmentation" International Journal of Innovative Research in Science,Engineering and Technology, Vol. 3, Issue 5, May 2014, pp.12360- 12368

[4] Supriya kale, AjinkyaShinde, SomsundarjiShinde, Prof. PallaviChandake, "Optical Character Recognisation”International Journal Of Modern Engineering Research, Vol. 5 Iss.4 Apr. 2015, pp.15-18

[5] NadiraMuda, NikKamariahNik Ismail, SitiAzami Abu Bakar, JasniMohamadZain, “Optical Character Recognition By Using Template Matching (Alphabet)"

[6] http://en.wikipedia.org/wiki/Template matching

[7] T. Mahalakshmi, R. Muthaiah and P. Swaminathan, "Review Article: An Overview of Template Matching Technique in Image Processing" Research Journal of Applied Sciences, Engineering and Technology, December 2012, ISSN: 2040-7467, pp. 5469-5473

[8] KavitaAhuja, PreetiTuli, “Object Recognition by Template Matching Using Correlations and Phase angle Method" International Journal of Advanced Research in Computer and Communication Engineering, Vol. 2, Issue 3, March 2013, ISSN (Print) : 2319-5940 ISSN (Online) : 2278-1021, pp. 1368- 1373.

[9] T. Kawanishi, T. Kurozumi, K. Kashino, S. Takagi, " A Fast Template Matching Algorithm with Adaptive Skipping Using Inner-Sub templates' Distances" Proc. a/the 17/h Inti. Con! On Pattern Recognition, vol. 3, pp. 654-657, Aug. (2004).

[10] D. Schonfeld, "On the relation of order-statistics filters and template matching: optimal morphological pattern recognition," IEEE Trans. on Image Process., vol. 9, no. 5, pp. 945-949, May. (2000).

[11] MohammmadGharavi-Alkhansari, "A Fast Globally Optimal Algorithm for Template Matching Using Low-Resolution Pruning" IEEE Trans. Image Processing, vol. 10, no. 4, pp. 526-533, Apr.(2001).

[12] Igor Guskov,"Kernel-based Template Alignment," Proc. a/IEEE Computer Society Conf on Computer vision and Pattern Recognition, pp. 610-617, (2006).

[13] Lawrence O'Gorman and RangacharKasturi “Document Image Analysis" ISBN 0-8186-7802-X

[14] Madhumathi.k, Dr.AntonySelvadossThanamani "Image Mining: frameworks and techniques" International Journal of Innovative Research in Computer and Communication Engineering, Vol.2, Special Issue 1, March 2014, ISSN(Online): 2320-9801, ISSN (Print): 2320-9798

[15] Wynne Hsu, Mong Li Lee and Ji Zhang "Image Mining: Trends and Developments" Journal of Intelligent Information Systems- 19:1, 7-23, 2002

[16] Ankit Kumar, Ashish Joshi, Anil Kumar, Ankush Mittal and D R Gangodkar "Template Matching Application In Geo-Referencing Of Remote Sensing Temporal Image" International Journal of Signal Processing (IJSIP), Image Processing and Pattern Recognition Vol.7, No.2 (2014), ISSN: 2005-4254 pp.201-210 http://dx.doi.org/10.14257/ijsip.2014.7.2.19

[17] Yufan Wang, Qiuze Yu, Wenxian Yu, -An Improved Normalized Cross Correlation algorithm for SAR Image Registration\|, IEEE IGARSS, 2012. 
[18] Vanderbrug, G.J, Rosenfeld, - Two-Stage Template Matching,|| IEEE Transactions on Computers, Vol. 60, Issue 11, 1977.

[19] C. Saravanan, M. Surender "Algorithm for Face Matching Using Normalized Cross-Correlation" International Journal of Engineering and Advanced Technology (IJEAT) ISSN: 2249 - 8958, Volume-2, Issue-4, April 2013

[20] By Douglas Lyon "The Discrete Fourier Transform, Part 6: Cross-Correlation" JOURNAL OF OBJECT TECHNOLOGY, Vol. 9, No. 2, March - April 2010, pp.17-22.

[21] Y. Raghavender Rao, Nikhil Prathapani, E.Nagabhooshanam "APPLICATION OF NORMALIZED CROSS CORRELATION TO IMAGE REGISTRATION” IJRET: International Journal of Research in Engineering and Technology eISSN: 2319-1163 | pISSN: 2321-7308

[22] J. B. Antoine Maintz and Max A. Viergever, "ASurvey of Medical Image Registration", Medical Image Analysis (1998) Volume2, number 1, pp 1-36, Oxford University Press.

[23] C. R. Maurer, G. B. Aboutanos, B. M. Dawant, R.A. Margolin, R. J. Maciunas and J. M. Fitzpatrick., "Registration of CT and MR brain images using a combination of points and surfaces", Medical imaging: image processing, volume 2434, Bellingham, WA, 1995. SPIE Press, 109-123.

[24] J. P. Lewis, Fast Normalized Cross-Correlation

[25] Duc Thanh Nguyen, Wanqing Li, Philip Ogunbona "A novel template matching method for human Detection" International Conference on Image Processing - 2009 (pp. 2549-2552). Cairo, Egypt: IEEE.

\section{AUTHORS}

\section{BIOGRAPHY}

Dr.S.Vijayarani, MCA, M.Phil, Ph.D., is working as Assistant Professor in the School of Computer Science and Engineering, Bharathiar University, Coimbatore. Her fields of research interest are data mining, privacy and security issues in data mining and data streams. She has published papers in the international journals and presented research papers in international and national conferences.

Ms. A. Sakila has completed M.Sc in Computer Science. She is currently pursuing her M.Phil in Computer Science in the Deaprtment of Computer Science, Bharathiar University, Coimbatore. Her field of research is image mining. She has published papers in international journals and presented papers in conferences.
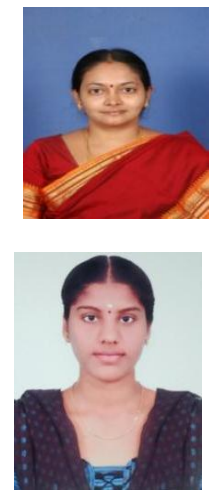